\title{
Mindfulness Based Cognitive Therapy (MBCT) as An Addition to Psychiatric Care as Usual for HIV-infected Patients with Mental Health Symptoms
}

\author{
Annemiek Schadé*, Gerard van Grootheest and Johannes H Smit \\ GGZ inGeest and Department of Psychiatry, EMGO Institute for Health and Care Research, VU University Medical Center, Amsterdam, the Netherlands
}

\begin{abstract}
Objectives: HIV-infected patients suffer from both physical and mental health symptoms and treatment of both symptoms is important. The 8-week Mindfulness Based Cognitive Therapy (henceforth MBCT) was originally developed for both mental and physical symptoms and is easy to implement. It is unknown, however, whether the addition of MBCT to psychiatric care as usual is effective in the long term and feasible for this group of patients. We measured depressive and anxiety symptoms, suicidal thoughts and fatigue in HIV-infected patients receiving psychiatric care as usual with or without MBCT, at intake and after one year.
\end{abstract}

Methods: The study was conducted at the outpatient clinic for HIV and Mental Health at GGZ inGeest in Amsterdam, the Netherlands, between March 2006 and September 2009. MBCT was offered as a group therapy in addition to individual care as usual to all $(\mathrm{N}=208)$ patients in their first year of psychiatric treatment.

Results: In the research period, 58 patients opted for the MBCT and 150 did not. Twenty patients did not complete the MBCT and questionnaires of both time points were available from 22 patients with and from 60 patients without MBCT. During their first year of psychiatric care, patients in both treatment groups showed significant improvement on depressive and anxiety symptoms, suicidal thoughts and fatigue.

Conclusion: Measured after one year, the addition of MBCT to psychiatric care as usual for HIV-infected patients had no measurable added effect on the treatment of depressive and anxiety symptoms, suicidal thoughts and fatigue. The present study was an explorative study to investigate the role of additional MBCT to psychiatric care as usual. It seems that general addition of MBCT is not feasible in this group of patients. However, further research, for example in a randomized controlled trial $(\mathrm{RCT})$ is necessary to confirm the results.

Keywords: Anxiety; Depressive symptoms; Fatigue; HIV; Mental health; Mindfulness based cognitive therapy (MBCT); Suicidal thoughts

\section{Introduction}

HIV-infected patients have an increased risk of mental health symptoms like depression, anxiety disorders, abuse of alcohol and drugs and personality disorders and there is a high prevalence of psychopathology in those patients [1-3]. In addition, suicidal ideations occur frequently and the risk of suicide and suicide attempts is high among HIV-infected patients, compared to the general population and compared to patients with other chronic somatic diseases [4].

Many HIV-infected patients suffer from pain and physical symptoms and there is a strong association between these symptoms and mental health symptoms [5]. HIV-related fatigue has a high prevalence and is strongly associated with psychological factors such as depression and anxiety [6].

Physical and mental health symptoms have a major negative influence on the quality of life and on the treatability, adherence, and prognosis of the HIV infection [2,7,8]. Both psychotherapy and medication have proven to be effective treatment for mental health problems in HIV-infected patients, especially with regard to depressive symptoms [9]. Therefore, it is important that treatments are developed that take both physical and mental aspects into account.

In the 1980s, such a combination program for chronically ill patients was developed by John Kabat-Zinn. He developed the Mindfulness Based Stress Reduction (MBSR) programme for patients with chronic pain and stress-symptoms based on meditation techniques and an alternative way of focussing attention [10]. Segal, Williams and Teasdale added elements of cognitive therapy and developed the Mindfulness Based Cognitive Therapy (henceforth MBCT) [11]. MBCT is a short, easy to realize 8-week therapy, consisting of 8 sessions with trained therapists. Patients are expected to do exercises and meditation at home for at least one hour per day.

Although the reported positive results have met with criticism [12], a meta-analysis showed that MBCT had small effects on depression, anxiety and psychological distress in people with chronic somatic diseases [13]. Randomized clinical trials (RCTs) have shown that combining MBCT with regular treatment significantly reduces the relapse risk in depressive symptoms or extends the time until relapse, compared with regular treatment alone $[11,14,15]$. Another study showed that MBCT reduced depressive symptoms and pain intensity and increased energy levels for patients with a traumatic brain injury and a clinically diagnosed depression [16].

*Corresponding author: Annemiek Schadé, Expert and Treatment Center on HIV and Mental Health, GGZ in Geest, VU University Medical Center, Amstelveenseweg 5891081 JC Amsterdam, The Netherlands, Tel: 00-31-20-7885000, E-mail: a.schade@ggzingeest.nl

Received January 25, 2016; Accepted February 15, 2016; Published February 23, 2016

Citation: Schadé A, van Grootheest G, Smit JH (2016) Mindfulness Based Cognitive Therapy (MBCT) as An Addition to Psychiatric Care as Usual for HIV-infected Patients with Mental Health Symptoms. J AIDS Clin Res 7: 547. doi:10.4172/2155 6113.1000547

Copyright: (C) 2016 Schadé A, et al. This is an open-access article distributed under the terms of the Creative Commons Attribution License, which permits unrestricted use, distribution, and reproduction in any medium, provided the original author and source are credited. 
Some research has been conducted into the effects of MBCT for HIV-infected patients [17]. The usefulness of MBCT has been tested in a few studies with divergent treatment outcomes, such as improvement of immunity, physical symptoms, side effects of antiretroviral therapy (ART), and mental health symptoms. These studies showed mixed results. One RCT only showed improvement in physical symptoms and not in CD4-count or mental health symptoms [18]. In another RCT, the largest effect was found for the reduction in the frequency of symptoms attributable to antiretroviral therapy (ART) [19]. In a nonrandomized study, the number and activity of natural killer cells increased significantly in the MBCT group compared to care as usual group [20]. However, no significant changes or differences were found for psychological, endocrine, or functional health variables. The studies of Duncan [19], Gayner [21] and Gonzales-Garcia [22] showed that MBT was marginally beneficial for relieving symptoms of depression in HIV-infected patients [17].

MBCT is a therapy with an intensive homework programme, consisting of exercises and meditation. During the training, the therapists discuss the homework and encourage the patients to do their exercises. However, it is important that patients implement mindfulness in their daily life, and not only during the research period. As most evaluations of the effectiveness of MBCT have been done almost immediately after the 8-week period, it is unclear whether the positive effect of MBCT in patients will last for a substantial period of time [23]. None of the studies mentioned above reported on the longterm effects (one year) of MBCT on mental health symptoms in HIVinfected patients and the feasibility of the addition of MBCT to care as usual. It remains unclear if supplementing regular treatment with MBCT constitutes a worthwhile addition.

We hypothesized that the general addition of MBCT to a psychiatric care as usual programme would show extra, long-term improvement on mental health symptoms and perceived lack of energy. The present study had one main objective. We tested if the general addition of Mindfulness Based Cognitive Therapy (MBCT) to psychiatric care as usual reduced depressive and anxiety symptoms, suicidal thoughts and fatigue in HIV-infected patients with mental health symptoms, measured one year after intake in a non-randomized study.

\section{Methods}

So far, most studies have investigated the effect of MBCT in an RCT. However, to our knowledge, no research has been published on the effect of the general addition of MBCT to psychiatric care as usual in a mental health treatment seeking HIV-infected population. Admittedly, a non-randomized study has disadvantages, but general addition of therapy is common in outpatient clinics. MBCT was not implemented in the out patient clinic and we did not know if it is feasible to offer MBCT to this group of patients and which group of patients would respond to the training. Therefore, the MBCT was offered to almost all patients and investigated in an open study with a prospective design.

We conducted the study at the outpatient clinic for HIV and Mental Health at GGZ inGeest in Amsterdam, the Netherlands, between March 2006 and September 2009. This clinic was founded in 1983 and specializes in the assessment and treatment of mental health symptoms like depression, anxiety disorder, personality disorder and use of alcohol and drugs among HIV-infected patients. Hundred and twenty new patients visit the clinic every year and can be referred by their infectious disease specialist, HIV-nurse, family doctor or regular psychologist or psychiatrist.
The study on general addition of Mindfulness Based Cognitive Therapy (MBCT)for HIV-infected patients with mental health problems was part of the study named 'HIV-infected mental health patients: characteristics and comparison with HIV-infected patients from the general population and non-infected mental health patients' [4].

New patients visiting the outpatient clinic were asked to participate in the study on HIV and mental health at their first appointment. The design and the purpose of the study were explained, and the patients gave written informed consent. At the intake, trained staff members obtained a DSM-IV diagnosis using the M.I.N.I. International Neuropsychiatric Interview (M.I.N.I. Plus) [24]. New patients were asked to fill in the assessment lists during their first appointment and, subsequently, after one year. Patients received up to three reminders if the questionnaire was not returned.

The Medical Ethics Review Committee of the VU University Medical Centre in Amsterdam approved the research protocol and all the patients who participated in the study gave written informed consent.

\section{Care as usual}

After diagnosis and treatment plan, all patients received supportive therapy or cognitive behaviour therapy from a psychologist, psychiatrist or a specialized mental health nurse. This care as usual was sometimes supplemented with psychotropic medication.

\section{Care as usual plus MBCT}

MBCT was offered as an additional treatment option to all patients in their first year of treatment. Exclusion criteria for participating in the MBCT groups were severe suicidality, depression, or psychosis to the extent that hospital admission was considered. Other exclusion criteria were unwillingness to do homework for the training and severe misbehaviour that would prevent functioning in a group. The MBCT groups consisted of up to 10 participants. There were two experienced trainers: a psychotherapist and a community mental health nurse. Both had been trained in delivering MBCT according to protocol and had experience giving the MBCT to patient groups prior to the current study. The MBCT was given to our patients by the same protocol, with eight weekly sessions of 2.5 hours and one follow up session after four weeks. On average, participants had to spend one hour per day on homework assignments.

\section{Measures}

Self-report questionnaires were used to obtain measures for depression, suicidality, anxiety and somatization. Additionally, the sum of Inventory of Depressive symptoms (IDS) items 20 (energy level) and 30 (feeling weighted down / physical energy) were used as an indicator of feeling insufficiently energetic. The following assessment lists were included:

Inventory of Depressive symptoms (IDS) [25]: The 30 item Inventory of Depressive Symptomatology (IDS) is designed to assess the severity of depressive symptoms.

The five screening items of the Beck Scale for Suicide Ideation (BSS) [26]: The BBS is a valuable tool for clinicians to examine suicidal intent in patients. Developed for use with patients of 17 years and over, the BSS provides a good starting point for a clinician's more detailed examination of a patient's suicidal intent.

Beck Anxiety Index (BAI) [27]: the Beck Anxiety Inventory (BAI) is a 21 -item self-report instrument that assesses the overall severity of 
Page 3 of 5

anxiety. The BAI was developed to address the need for an instrument that would reliably discriminate anxiety from depression while displaying convergent validity.

Four Dimensional Symptom Questionnaire (4DSQ) [28]: The somatization scale is one of the 4 scales of the Four-Dimensional Symptom Questionnaire, a self-report questionnaire designed to assess common psychological symptoms in primary care. The Somatization scale measures a range of common physical symptoms, known to be related to distress or psychopathology. Examples are headache, palpitations, nausea, and muscle aches.

\section{Analyses}

First, we tested whether patients who opted for MBCT were any different from those who did not, in terms of demographics, symptom severity or DSM-IV diagnosis. We used ANOVA to compare group means of continuous variables, and the Pearson chi-square statistic to test for differences between groups on discrete variables. When cells had an expected count smaller than 5 , the likelihood ratio is reported instead of the Pearson chi-square statistic.

Paired t-tests and chi-square tests were used among completers, in order to test whether patients in either group showed any change of symptoms one year after intake. In a third analysis, we used ANCOVA and chi-square tests to test whether the absolute change of symptom scores differed between the two treatment groups. Patients who dropped out of the MBCT group were not included in any of these analyses. Data were analysed using SPSS Statistics 20 (IBM Corp., Armonk, NY, USA).

\section{Results}

Between 2006 and 2009, the therapy was offered to 58 patients in total. Of these patients 38 filled in the baseline questionnaire and completed the training within their first year after intake and 22 patients returned the assessment list one year after intake. In the same period, another 150 patients had an intake at the mental health clinic and filled in the questionnaire, but did not participate in the MBCT. Of this group, who only received care as usual, 60 patients responded to the questionnaire after one year.

The patients who followed the MBCT were older and had been HIV-positive for a longer time than the patient in the care as usual (CAU) only group. Their mean CD4 count was also higher. Patients in the MBCT group were less frequently diagnosed with drug use disorders, but did not differ from the CAU-only patients when it came to other DSM diagnoses or psychiatric symptom severity. The most prevalent diagnoses both groups shared were depressive and anxiety disorders (Table 1).

\section{Effect of treatment after one year}

Both the CAU-only and the CAU + MBCT patients showed a significant improvement in terms of depressive and anxiety symptoms and perceived fatigue during the one-year period after intake (Table 2). Furthermore, suicidal ideation decreased significantly in the CAU + MBCT-group, while reaching no significance in the CAU condition. 4DSQ scores did not improve significantly in either condition.

We furthermore tested whether the improvement of depressive and anxiety symptoms was greater in one group than the other. Table 3 shows the mean change in symptom severity during the one-year period following intake. ANCOVA analysis was used to correct for any differences between groups on symptom scores at intake. The addition of MBCT did not lead to an improvement that was better than CAU-

\begin{tabular}{|l|c|c|c|}
\hline & $\begin{array}{c}\text { CAU-only } \\
(\mathbf{N}=150)\end{array}$ & $\begin{array}{c}\text { CAU + MBCT } \\
\mathbf{( N = 3 8 )}\end{array}$ & Significance \\
\hline Sex & $89 \%$ male & $82 \%$ male & $0.261^{2}$ \\
\hline Mean age & $40.6( \pm 8.8)$ & $46.7( \pm 7.1)$ & $<0.001^{1 \star}$ \\
\hline Mean years of HIV & $6.0( \pm 5.7)$ & $10.4( \pm 7.3)$ & $<0.001^{1 *}$ \\
\hline Mean CD4 count & $468( \pm 215)$ & $591( \pm 299)$ & $0.013^{1 *}$ \\
\hline CD4<200 & $6 \%$ & $7 \%$ & $0.711^{2}$ \\
\hline Mean IDS & $29.3( \pm 13.6)$ & $29.4( \pm 11.8)$ & $0.992^{1}$ \\
\hline Mean BAI & $17.4( \pm 11.9)$ & $16.2( \pm 8.8)$ & $0.563^{1}$ \\
\hline Mean 4DSQ & $13.3( \pm 10.2)$ & $13.7( \pm 9.4)$ & $0.842^{1}$ \\
\hline Mean lack of energy & $2.73( \pm 1.58)$ & $3.05( \pm 1.43)$ & $0.250^{1}$ \\
\hline Suicide ideation & $46 \%$ & $49 \%$ & $0.810^{3}$ \\
\hline Drug used last month & $47 \%$ & $29 \%$ & $0.068^{3}$ \\
\hline DSM diagnosis axis 1 & & & \\
\hline Depression & $51 \%$ & $53 \%$ & $0.829^{3}$ \\
\hline Anxiety & $25 \%$ & $13 \%$ & $0.128^{3}$ \\
\hline Alcohol dependence / abuse & $8 \%$ & $8 \%$ & $0.983^{2}$ \\
\hline Drugs dependence / abuse & $15 \%$ & $3 \%$ & $0.016^{2 \star}$ \\
\hline Bipolar or psychotic disorders & $1 \%$ & $5 \%$ & $0.180^{2}$ \\
\hline Adjustment disorders & $11 \%$ & $8 \%$ & $0.603^{2}$ \\
\hline${ }^{2}$ ANOVA & & & \\
\hline Likelihood ratio & & & \\
\hline${ }^{2}$ Pearson chi-square & & & \\
\hline
\end{tabular}

Table 1: Mental health symptoms and demographic characteristics at intake.

\begin{tabular}{|c|c|c|c|}
\hline & Intake & 1 year & Significance \\
\hline \multicolumn{4}{|l|}{ CAU-only (N=60) } \\
\hline Mean IDS & $29.6( \pm 13.4)$ & $25.5( \pm 14.6)$ & $0.003^{1 *}$ \\
\hline Mean BAI & $18.5( \pm 12.5)$ & $13.2( \pm 11.1)$ & $0.001^{1 *}$ \\
\hline Mean 4DSQ & $14.0( \pm 10.3)$ & $13.1( \pm 11.8)$ & $0.429^{1}$ \\
\hline Mean lack of energy & $3.04( \pm 1.59)$ & $2.49( \pm 1.75)$ & $0.011^{1 *}$ \\
\hline Suicide ideation & $53 \%$ & $34 \%$ & $0.051^{2}$ \\
\hline \multicolumn{4}{|l|}{ CAU+MBCT $(\mathrm{N}=22)$} \\
\hline Mean IDS & $33.5( \pm 11.8)$ & $23.9( \pm 10.7)$ & $0.002^{1 *}$ \\
\hline Mean BAI & $18.6( \pm 9.2)$ & $13.6( \pm 9.3)$ & $0.014^{1 *}$ \\
\hline Mean 4DSQ & $14.7( \pm 10.2)$ & $11.5( \pm 7.8)$ & $0.056^{1}$ \\
\hline Mean lack of energy & $3.55( \pm 1.41)$ & $2.68( \pm 1.58)$ & $0.038^{1 *}$ \\
\hline Suicide ideation & $57 \%$ & $23 \%$ & $0.021^{2 *}$ \\
\hline
\end{tabular}

Table 2: Symptom severity at intake and after one year.

\begin{tabular}{|l|c|c|c|}
\hline & $\begin{array}{c}\text { CAU-only } \\
(\mathbf{N}=\mathbf{6 0})\end{array}$ & $\begin{array}{c}\text { CAU + MBCT } \\
\mathbf{( N = 2 2 )}\end{array}$ & Significance \\
\hline$\Delta$ IDS & $-4.2( \pm 10.0)$ & $-9.6( \pm 12.6)$ & $0.101^{1}$ \\
\hline$\Delta$ BAI & $-5.3( \pm 12.1)$ & $-4.9( \pm 8.6)$ & $0.846^{1}$ \\
\hline$\Delta 4 \mathrm{DSQ}$ & $-0.9( \pm 8.9)$ & $-3.2( \pm 7.5)$ & $0.301^{1}$ \\
\hline$\Delta$ Lack of energy & $-0.42( \pm 1.6)$ & $-0.86( \pm 1.8)$ & $0.819^{1}$ \\
\hline Suicide ideation & $7.5 \%$ & $4.8 \%$ & \\
\hline Increased ideation & $69.8 \%$ & $57.1 \%$ & $0.406^{2}$ \\
\hline No change & $22.6 \%$ & $38.1 \%$ & \\
\hline Decreased ideation & $73 \%$ & $71 \%$ & $0.886^{3}$ \\
\hline Received treatment after one year & \multicolumn{3}{|l}{} \\
\hline $\begin{array}{l}\text { 1ANCOVA (correcting for scores at intake) } \\
\text { 2Likelihood ratio }\end{array}$ & & \\
\hline
\end{tabular}

Table 3: Effects of treatment condition on symptom change during the first year after intake.

only on any of the symptom scores. Indeed, this result persisted when age was added as a covariate. Also, the number of patients who still received treatment at the mental health clinic after one year did not differ between groups. 


\section{Discussion}

HIV-infected patients often suffer from both mental and physical symptoms [5]. Treatment of both mental and physical problems improves quality of life and the prognosis of the HIV infection [29].

The effect of MBCT for patients with HIV and mental health symptoms has been documented in several studies [17]. Most research is carried out in an RCT with a control group. To our knowledge, our research project is the first on HIV-infected patients with mental health problems who received treatment for their mental health problems and were offered MBCT as an addition to their treatment. Since MBCT was an additional voluntary treatment option, patients who joined the therapy group were motivated for the therapy and were willing to do their homework. Although no randomization had taken place, there were no important differences at baseline between the two groups of patients (CAU-only and CAU + MBCT, respectively). In the CAU + MBCT group, the patients were older and therefore they had been longer infected with HIV. It seems the older patients were more willing to invest in homework and meditation exercises. In a previous study we already determined that older HIV-infected patients did not have more depressive or anxious symptoms, suicidal ideation and suicide attempts [4]. The CAU-only group used more drugs, but did not have a different baseline score on depressive and anxiety symptoms, suicidal thoughts, or fatigue.

There was a significant improvement of patients who received MBCT on depressive and anxiety symptoms, suicidal thoughts and fatigue measured one year after intake. However, the group of patients who only received CAU also showed significant improvement. One year after intake, there was no important significant difference between the two groups. Both groups of patients improved significantly after one year of treatment. The effect of the extra treatment option of MBCT was not measurable in our chosen treatment outcome. Therefore, we might conclude that in our study the general addition of MBCT to psychiatric care as usual for HIV-infected patients had no long-term added value.

Different reasons may explain why no additional effect of MBCT was found in the present study. There may have been a positive effect just after the MBCT, but there was no similar assessment of the CAUonly group in order to compare the two groups. It is unclear if the patients with the extra MBCT implemented the therapy in their daily life, or stopped doing the exercises after the therapy was finished. Alternatively, it is possible that the addition of MBCT to psychiatric care as usual has no positive effect at all on HIV-infected patients with mental health symptoms. The finding that only about one-third of new patients were able or wanted to participate in the training and about one- third of the participants did not finish the training indicates that general addition of MBCT may not be feasible in this group of patients.

To examine the exclusive effect of MBCT, a design with MBCT and an other group training is essential. Especially HIV-infected patients, who join a group with other HIV-infected patients can benefit from the presence of other group members with the possible result that they feel more understood and suffer less from stigmatization which can improve quality of life.

\section{Limitations of the Study}

This study has several limitations. Firstly, we did not study the addition of MBCT according to the gold standard of an RCT. Secondly, the number of patients who received MBCT and completed the assessment lists is limited. Thirdly, there was only one assessment at intake and one assessment at the follow up one year later from the group of patients who received care as usual only. Therefore, it was not possible to compare the groups after, say, six months of treatment with the group who received care as usual with MBCT. Fourthly, we did not measure 'quality of life', an important issue in HIV-infected patients. MBCT might have contributed to the improvement of quality of life, but that was not measured.

\section{Conclusion}

One year after intake there was no positive effect of the additional MBCT to psychiatric care when compared to care as usual alone. Both groups of patients improved significantly one year after intake on depressive and anxiety symptoms, suicidal thoughts and fatigue. Assessed after one year, the addition of MBCT to psychiatric care as usual in this population showed no measurable added value. It seems that general addition of MBCT is not feasible in this group of patients. However, future research on MBCT in HIV-positive patients with mental health symptoms is needed in a RCT with multiple assessments and measuring 'quality of life'.

\section{References}

1. Alegria M, Vila D, Train S, Williams S, El-Bassel N (2006) Psychiatric aspects of HIVIAIDS. ILippincott, Williams \& Wilkens.

2. Chander G, Himelhoch S, Moore D (2006) Substance abuse and psychiatric disorders in HIV-positive patients: epidemiology and impact on antiretroviral therapy. Drugs 66: 769-789.

3. Lopes M, Olfson M, Rabkin J, Hasin DS, Alegría AA, et al. (2012) Gender, HIV status, and psychiatric disorders: results from the National Epidemiologic Survey on Alcohol and Related Conditions. J Clin Psychiatry 73: 384-391.

4. Schadé A, van Grootheest G, Smit JH, Smit JH (2013) HIV-infected mental health patients: characteristics and comparison with HIV-infected patients from the general population and non-infected mental health patients. BMC Psychiatry 13: 35.

5. Merlin JS, Cen L, Praestgaard A, Turner M, Obando A, et al. (2012) Pain and physical and psychological symptoms in ambulatory HIV patients in the current treatment era. J Pain Symptom Manage 43: 638-645.

6. Jong E, Oudhoff LA, Epskamp C, Wagener MN, van Duijn M, et al. (2010) Predictors and treatment strategies of HIV-related fatigue in the combined antiretroviral therapy era. AIDS 24: 1387-1405.

7. Ickovics JR, Hamburger ME, Vlahov D, Schoenbaum EE, Schuman P, et al. (2001) Mortality, CD4 cell count decline, and depressive symptoms among HIV-seropositive women: longitudinal analysis from the HIV Epidemiology Research Study. JAMA 285: 1466-1474.

8. Johnson MO, Stallworth T, Neilands TB (2003) The drugs or the disease? Causal attributions of symptoms held by HIV-positive adults on HAART. AIDS Behav 7: 109-117.

9. Olatunji BO, Mimiaga MJ, O'Cleirigh C, Safren SA (2006) Review of treatment studies of depression in HIV. HIV Med 14: 112-124.

10. Kabat-Zinn J, Lipworth L, Burney R (1985) The clinical use of mindfulness meditation for the self-regulation of chronic pain. J Behav Med 8: 163-190.

11. Teasdale JD, Segal ZV, Williams JMG, Ridgeway VA, Soulsby JM, et al. (2000) Prevention of relapse/recurrence in major depression by mindfulness-based cognitive therapy. Journal of Consulting and Clinical Psychol 68: 615-623.

12. Havermans RC (2011) Werkt mindfulnessmeditatietherapie nu echt, of echt niet? Psychologie \& Gezondheid 39: 42-46.

13. Bohlmeijer E, Prenger R, Taal E, Cuijpers P (2010) The effects of mindfulnessbased stress reduction therapy on mental health of adults with a chronic medical disease: a meta-analysis. J Psychosom Res 68: 539-544.

14. Ma SH, Teasdale JD (2004) Mindfulness-based cognitive therapy for depression: replication and exploration of differential relapse prevention effects. Journal of Consulting and Clinical Psychology 72: 31-40.

15. Godfrin, K, van Heeringen C (2010) The effects of mindfulness-based cognitive therapy on recurrence of depressive episodes, mental health and quality of life: A randomized controlled study. Behavioral Research and Therapy 48: 738-746. 
Citation: Schadé A, van Grootheest G, Smit JH (2016) Mindfulness Based Cognitive Therapy (MBCT) as An Addition to Psychiatric Care as Usual for HIV-infected Patients with Mental Health Symptoms. J AIDS Clin Res 7: 547. doi:10.4172/2155-6113.1000547

16. Bédard M, Felteau M, Marshall S, Campbell S, Gibbons C, et al. (2012) Mindfulness-based cognitive therapy: benefits in reducing depression following a traumatic brain injury. Advances in Mind-Body Medicine 26: 14-20.

17. Yang Y, Yan-Hui L, Hong-Fu Z, Jing-Ying J (2015) Effectiveness of mindfulnessbased stress reduction and mindfulness-based cognitive therapies on people living with HIV: A systematic review and meta-analysis. International Journal of Nursing Sciences 2: 283-294.

18. SeyedAlinaghi S, Jam S, Foroughi M, Imani A, Mohraz M, et al. (2012) Randomized controlled trial of mindfulness-based stress reduction delivered to human immunodeficiency virus-positive patients in Iran: effects on CD4*T lymphocyte count and medical and psychological symptoms. Psychosomatic Medicine 74: 620-6277.

19. Duncan LG, Moskowitz JT, Neilands TB, Dilworth SE, Hecht FM, et al. (2012) Mindfulness-based stress reduction for HIV treatment side effects: a randomized, wait-list controlled trial. J Pain Symptom Manage 43: 161-171.

20. Robinson FP, Mathews HL, Witek-Janusek L (2003) Psycho-Endocrine-Immune Response to Mindfulness-Based Stress Reduction in Individuals Infected with the Human Immunodeficiency Virus: A Quasiexperimental Study. Journal of Alternative and Complementary Medicine 9: 683-694.

21. Gayner B, Esplen M, DeRoche P, Bishop S, Kavanagh L, et al. (2012) A Randomized controlled trial of mindfulness-based stress reduction to manage affective symptoms and improve quality of life in gay men living with HIV. J Behav Med 35: 272-285.

22. Gonzalez-Garcia M, Ferrer MJ, Borras X, Muñoz-Moreno JA, Miranda C, et al.
(2013) Effectiveness of Mindfulness-Based Cognitive Therapy on the Quality of Life, Emotional Status, and CD4 Cell Count of Patients Aging with HIV Infection. AIDS Behav.

23. Grossman P, Niemann L, Schmidt S, Walach H (2004) Mindfulness-based stress reduction and health benefits. A meta-analysis. J Psychosom Res 57: 35-43.

24. Vliet van IM, Leroy H, Van Megen HJGM (2000) M.I.N.I. Internationa Neuropsychiatric Interview; Dutch version 5.0.0.R. Department of Psychiatry University Medical Center Utrecht, The Netherlands.

25. Rush AJ, Giles DE, Schlesser MA, Fulton CL, Weissenburger J, et al. (1986) The Inventory for Depressive Symptomatology (IDS): preliminary findings. Psychiatry Res 18: 65-87.

26. Beck AT, Steer RA, Ranieri WF (1988) Scale for Suicide Ideation: psychometric properties of a self-report version. J Clin Psychol 44: 499-505.

27. Beck AT, Epstein N, Brown G, Steer RA (1988a) An inventory for measuring clinical anxiety: Psychometric properties. Journal of Consulting and Clinical Psychology 56: 893-897.

28. Terluin B, Van Marwijk HWJ, Adèr $\mathrm{HJ}$, De Vet HCW, Penninx BWJH, et al. (2006) The Four-Dimensional Symptom Questionnaire (4DSQ): a validation study of a multidimensional self-report questionnaire to assess distress, depression, anxiety and somatization. BMC Psychiatry 6: 34-40.

29. Sherbourne CD, Hays RD, Fleishman JA, Vitiello B, Magruder KM, et al. (2000) Impact of psychiatric conditions on health-related quality of life in persons with HIV infection. Am J Psychiatry 157: 248-254. 